\title{
Which Sections of Electronic Medical Records Are Most Relevant for Real-Time Surveillance of Influenza- like Illness?
}

\author{
Dino P. Rumoro', Shital C. Shah ${ }^{1}$, Marilyn M. Hallock¹, Gillian S. Gibbs ${ }^{* 1}$, Gordon M. \\ Trenholme ${ }^{1}$, Michael J. Waddell ${ }^{2}$ and Joseph P. Bernstein ${ }^{3}$
}

${ }^{1}$ Rush University, Chicago, IL, USA; ${ }^{2}$ Pangaea Information Technologies, Ltd, Chicago, IL, USA; ${ }^{3} \mathrm{H}-\mathrm{CORE}$, LLC, Chicago, IL, USA

\section{Objective}

To investigate which section(s) of a patient's electronic medical record (EMR) contains the most relevant information for timely detection of influenza-like illness (ILI) in the emergency department (ED).

\section{Introduction}

Effective real-time surveillance of infectious diseases must strike a balance between reliability and timeliness for early detection. Traditional syndromic surveillance utilizes limited sections of the EMR, such as chief complaints and/or diagnosis. However, other sections of the EMR may contain more pertinent information than what is captured in a brief chief complaint. These other EMR sections may provide relevant information earlier in the patient encounter than at the diagnosis or disposition stage, which can appear in the EMR up to 24 hours after the patient's discharge. Comprehensive analysis may identify the most relevant section of EMRs for surveillance of all major infectious diseases, including ILI.

\section{Methods}

This was a retrospective, cross-sectional study. The sample consisted of 100 randomly selected ED ILI-positive patients at an academic medical center. These patients came to the emergency department during the 2014-2015 ILI season (September 1, 2014 to April 30,2015). Geographic Utilization of Artificial Intelligence in Real-Time for Disease Identification and Alert Notification (GUARDIAN) - a syndromic surveillance program - was used to identify the positive ILI patients by applying the Centers for Disease Control and Prevention case definition of ILI (i.e., fever with cough and/or sore throat) to the entire EMR.

For each patient, the presence or absence of each ILI symptom was documented by a board-certified emergency physician for each section of the EMR, specifically: registration/arrival complaints, triage chief complaints, flow sheet/ vital signs data, history of present illness (HPI), review of systems (ROS), physical exam, assessment plan, diagnosis, free-text clinical notes, and discharge instructions, among others. In addition, efficacy of each EMR section in detecting ILI was documented.

\section{Results}

The ILI symptoms documented in the HPI section of the EMR captured $80 \%$ of ILI cases (Table 1). Thirty-nine percent of ILI cases had ILI symptoms documented in registration arrival complaints/ screening questions/triage chief complaints, while $91 \%$ of ILI cases had symptoms listed in the free-text sections of ROS and/or HPI plus flowsheet vital signs. In addition, only $46 \%$ of ILI cases had ILI symptoms documented in the discrete data fields of the EMR.

\section{Conclusions}

The HPI, ROS, and nursing notes sections of the EMR were information rich and the most relevant sections for ILI surveillance. Since $61 \%$ of cases reported ILI symptoms in areas of the EMR other than the commonly-used triage and registration sections, it is warranted that expanding ED syndromic surveillance to other areas of the EMR may increase sensitivity. Thus, reliable real-time syndromic surveillance systems need to be capable of processing both discrete and free-text data from various sections of the EMR.

Table 1: Percent of each section of EMR that meets the definition of ILI

\begin{tabular}{|c|c|}
\hline Sections(s) of EMR & $\begin{array}{c}\text { \% ILI Cases } \\
(\mathbf{n = 1 0 0})\end{array}$ \\
\hline History of present illness (HPI) & $\mathbf{8 0 \%}$ \\
\hline Review of systems (ROS) & $\mathbf{7 0 \%}$ \\
\hline Nurses & $\mathbf{4 6 \%}$ \\
\hline Attending notes & $\mathbf{3 2 \%}$ \\
\hline Assessment plan & $\mathbf{2 4 \%}$ \\
\hline Screening questions & $\mathbf{2 0 \%}$ \\
\hline Triage chief complaint (CC) & $\mathbf{1 8 \%}$ \\
\hline Registration arrival complaints & $\mathbf{8 \%}$ \\
\hline Diagnoses & $\mathbf{3 \%}$ \\
\hline Physical exam & $\mathbf{1 \%}$ \\
\hline Residents & $\mathbf{1 \%}$ \\
\hline Others & $\mathbf{1 \%}$ \\
\hline Nurse practitioner (NP) notes & $\mathbf{1 \%}$ \\
\hline Flowsheet vital signs & $\mathbf{0 \%}$ \\
\hline Flowsheet vital signs \& HPI \& ROS & $\mathbf{9 1 \%}$ \\
\hline HPI \& ROS & $\mathbf{8 1 \%}$ \\
\hline Registration arrival complaints \& Screening questions \& Triage CC \& & $\mathbf{4 6 \%}$ \\
\hline Flowsheet vital signs \\
\hline Registration arrival complaints \& Screening questions \& Triage CC & $\mathbf{3 9 \%}$ \\
\hline Attending \& Resident \& NP Notes & $\mathbf{3 4 \%}$ \\
\hline
\end{tabular}

Keywords

Influenza-like illness; GUARDIAN; Electronic Medical Records

\section{Acknowledgments}

GUARDIAN is funded by the US Department of Defense, Telemedicine and Advanced Technology Research Center, Award numbers W81XWH-09-1-0662 and W81XWH-11-1-0711.

\section{*Gillian S. Gibbs}

E-mail: Gillian_Gibbs@rush.edu 